\title{
Association between continuity of care in general practice and hospital admissions for ambulatory care sensitive conditions: cross sectional study of routinely collected, person level data
}

\author{
Isaac Barker, Adam Steventon, Sarah R Deeny
}

Data Analytics, The Health

Foundation, London WC2E 9RA,

UK

Correspondence to: I Barker isaac.barker@health.org.uk

Cite this as: BMJ 2017;356:j84 http://dx.doi.org/10.1136/bmj.j84

Accepted: 21 December 2016

\author{
ABSTRACT \\ OBJECTIVE \\ To assess whether continuity of care with a general \\ practitioner is associated with hospital admissions for \\ ambulatory care sensitive conditions for older \\ patients.
}

DESIGN

Cross sectional study.

SETTING

Linked primary and secondary care records from 200 general practices participating in the Clinical Practice Research Datalink in England.

PARTICIPANTS

230472 patients aged between 62 and 82 years and who experienced at least two contacts with a general practitioner between April 2011 and March 2013.

MAIN OUTCOME MEASURE

Number of hospital admissions for ambulatory care sensitive conditions (those considered manageable in primary care) per patient between April 2011 and March 2013.

\section{RESULTS}

We assessed continuity of care using the usual provider of care index, which we defined as the proportion of contacts occurring between April 2011 and March 2013 that were with the most frequently seen general practitioner. On average, the usual provider of care index score was 0.61 . Continuity of care was lower among practices with more doctors (average score 0.59 in large practices versus 0.70 in small practices). Higher continuity of care was associated with fewer admissions for ambulatory care sensitive conditions. When modelled, controlling for demographic and clinical patient characteristics, an

\section{WHAT IS ALREADY KNOWN ON THIS TOPIC}

To decrease unplanned hospital admissions, many healthcare systems have

focused on improving speed and equity of access to primary care

Continuity of care has been falling in England, perhaps because there are trade-offs between promoting access and continuity

Continuity of care is associated with patient and practitioner satisfaction, but its link with hospital admissions has been unclear

\section{WHAT THIS STUDY ADDS}

Continuity of care is associated with lower hospital admissions for ambulatory care sensitive conditions, which are those considered manageable in primary care

For heavy users of general practice, the association between continuity of care and hospital admissions for ambulatory care sensitive conditions is clearest

Strategies that improve continuity of care might simultaneously improve quality and reduce cost, although interventions will require evaluation

increase in the usual provider of care index of 0.2 for all patients would reduce these admissions by $6.22 \%$ (95\% confidence interval $4.87 \%$ to $7.55 \%$ ). There was greater evidence for an association among patients who were heavy users of primary care. Heavy users also experienced more admissions for ambulatory care sensitive conditions than other patients $(0.36$ admissions per patient for those with $\geq 18$ contacts with a general practitioner, compared with 0.04 admissions per patient for those with 2-4 contacts).

\section{CONCLUSIONS}

Strategies that improve the continuity of care in general practice may reduce secondary care costs, particularly for the heaviest users of healthcare. Promoting continuity might also improve the experience of patients and those working in general practice.

\section{Introduction}

Healthcare systems around the world are facing increases in unplanned hospital admissions. ${ }^{1}$ These admissions are often undesirable for patients, disruptive of elective care, and costly, so their prevention has become a priority. A large proportion of hospital admissions could be avoided through effective management and treatment in the community, ${ }^{2}$ which has become a focus of initiatives such as the accountable care organisations in the United States and the "five year forward view" strategy in the National Health Service in England. ${ }^{34}$ The focus has often been on improving access to primary care (for example, by increasing opening hours or introducing remote care)..$^{5-7}$ These initiatives have achieved mixed impacts on patient outcomes, ${ }^{8}$ and some may have had unintended effects on the continuity of the care provided. ${ }^{910}$

Although timely access is an important goal for high quality healthcare, ${ }^{11}$ continuity of care is associated with patient satisfaction, ${ }^{12} 13$ as well as quality of life for patients living with long term conditions. ${ }^{14}$ It encompasses several aspects, including the consistency of care with a healthcare professional over time, quality of the interpersonal relationships between healthcare professionals and patients, and availability of information about the patient. ${ }^{12}$ General practitioners value continuity of care, often considering it to be a core value of their profession, ${ }^{15} 16$ while many patients value a personal doctor to coordinate and integrate their care. ${ }^{1217}$ Unfortunately, there are indications from patient surveys that continuity of care is in decline in both the United States and England, ${ }^{1819}$ at a time when doctors are reporting concerns about their ability to provide coordinated care for the increasing number of 
patients with long term conditions. ${ }^{6}$ Part of the background is a trend towards larger general practices, where larger clinical and support teams manage a bigger patient list. ${ }^{20}$ Although larger practices are associated with improved quality in some respects, such as following good practice in chronic disease management and offering longer consultation times, ${ }^{2122}$ patients in larger practices report lower satisfaction with several aspects of care, including continuity. ${ }^{23}$

Although concerns have been raised about continuity, ${ }^{9}$ it is unclear how much effort and resource should be spent in tackling these compared with other improvement priorities such as access. Part of the problem is that few studies have examined whether continuity of care is associated with unplanned admissions, and the existing studies have major limitations. None have examined the relative benefit of continuity of care to different patient groups, including those with differing patterns of primary care utilisation. ${ }^{24}$ In one case, this is because survey data were aggregated to the general practice level, ${ }^{24}$ with the additional limitations and bias associated with practice level analysis. ${ }^{2425}$ Other studies have examined all cause unplanned admissions rather than the specific conditions most likely to be preventable by good quality primary care. ${ }^{1026-28}$ In the absence of robust evidence, the link between continuity and admissions can only be conjectured. One theory is that continuity might improve the management of long term conditions by increasing the ability of doctors to respond to patients' needs and preferences and therefore their ability to recommend suitable and acceptable courses of treatment. Continuity might also enable earlier detection and treatment of acute events, ${ }^{29}$ or improve the relationship between the general practitioner and patient and thus increase the uptake of preventive interventions such as vaccination or routine drug review, ${ }^{30}$ although this relationship is necessarily complex. Since some of these mechanisms might increase rather than reduce secondary care utilisation, ${ }^{31}$ more robust evidence is needed regarding the association between continuity of care in general practice and hospital admissions.

In this study, we use person level data to assess the relationship between continuity of care and hospital admissions, and examine how the association differs between high and low users of primary care. We address hospital admissions for ambulatory care sensitive conditions, which are (at least in theory) manageable in primary care. ${ }^{3233}$ Ambulatory care sensitive conditions include long term conditions, such as asthma, where good quality care should prevent episodes of flare-ups; acute conditions, such as gangrene, where timely and effective care stops the condition developing; and conditions that are preventable by vaccination, such as influenza and pneumonia. Unplanned admissions for these conditions accounted for $€ 1.42 \mathrm{bn}$ (\$1.74bn; €1.67) of spending in England in 2009/10.34 We focused our study on older patients (aged 62-82 years), who particularly value continuity of care and experience more admissions for ambulatory care sensitive conditions than the general population. ${ }^{34}$ One of the benefits of our study is that, as with some previous research, ${ }^{1031-38}$ we have used person level data from the electronic medical record in primary care to measure continuity of care. These data are increasingly available for use in quality improvement and can be assembled in a timely fashion at minimal cost. Thus measurement strategies based on these data have widespread applicability. If these indices show a link with patient outcomes that can be improved through intervention in primary care, then they could support quality improvement activities.

\section{Methods \\ Dataset}

This was a retrospective, observational, and cross sectional study using data from the Clinical Practice Research Datalink, which contains the electronic medical records of participating general practices, and includes contacts with healthcare professionals, details of diagnostic tests, referrals to specialist healthcare, diagnosed health conditions, personal characteristics, and the start and end dates of patient registrations with general practices. ${ }^{39}$ Patients in the practices participating in the Clinical Practice Research Datalink are broadly representative of patients registered to practices in England. ${ }^{40}$

The dataset was pseudonymised (all patient identifiable data had been removed) but linked at the person level to the hospital episode statistics, a national database containing administrative records from all hospitals in England that provide NHS care. From that database we obtained dates of inpatient admission and details of the diagnoses recorded during the inpatient stay (based on international classification of diseases, 10th revision, or ICD-10, codes). We also obtained and linked data on deaths occurring inside or outside of hospital from the Office for National Statistics, and socioeconomic deprivation scores for the small area of individual patient residence (index of multiple deprivation 2010).

\section{Inclusion and exclusion criteria}

We studied patients registered at general practices that participated in the Clinical Practice Research Datalink and that supplied data that met a minimum standard of quality. We included patients who were aged between 62 and 82 on 1 April 2011 and experienced at least two contacts with a general practitioner between April 2011 and March 2013-the period analysed in the study. To allow for a minimum of two years' follow-up for every patient, we excluded those who died before March 2013. We also required that patients were registered with a Clinical Practice Research Datalink practice for at least one year before April 2011, so that we could derive the study covariates. Finally, we excluded a small number of patients whose age, index of multiple deprivation score, or sex was not recorded.

Admissions for ambulatory care sensitive conditions Our outcome was the number of inpatient admissions for ambulatory care sensitive conditions for each patient between April 2011 and March 2013. Although several similar definitions of ambulatory care sensitive 
admissions exist, ${ }^{32}$ after a review of the literature we chose a core set of 22 such conditions, which we adapted from Bardsley et $\mathrm{al}^{32}$ (see supplementary appendix 1). Using the recorded ICD-10 codes we identified the corresponding admissions from the hospital episode statistics data. For some conditions (eg, asthma) we limited our search to the primary diagnosis field, whereas for others (eg, gangrene) we included all fields (see supplementary appendix 1).

\section{Continuity of care}

Continuity of care is a complex, multifaceted concept reflecting at least four domains ${ }^{12}$ : the subjective experience of a caring relationship (interpersonal continuity), a history of interacting with the same healthcare professional across a series of discrete episodes (longitudinal continuity), the availability of clinical and psychosocial information across encounters and professionals (informational continuity), and the effective collaboration of teams across care boundaries to provide seamless care (management continuity). In this study, we dealt with longitudinal continuity of care, which we measured using the usual provider of care index. Usual provider of care is defined as the proportion of a patient's contacts that was with their most regularly seen doctor. For example, if a patient had 10 general practitioner contacts, including six with the same doctor, then their usual provider of care index score would be 0.6 . We measured the usual provider of care index score for April 2011 to March 2013, the same period used for hospital admissions.

\section{Study covariates}

For each patient we determined age, sex; socioeconomic deprivation score grouped into fifths (based on national thresholds, with the first fifth representing the least deprived), ${ }^{41}$ number of contacts with a general practitioner (April 2011 to March 2013), number of active previous long term health conditions (recorded before April 2011), and number of previous referrals to specialist care (in 2010/11).

We identified contacts with general practitioners from the Clinical Practice Research Datalink data by selecting records corresponding to specific types of encounter (eg, clinic, home visit, telephone) and which were entered by general practitioners (including partners, registrars, sole practitioners, and locum doctors). All contacts were linked to a specific practitioner. Contacts with a general practitioner were limited to one each day, owing to concerns about duplication. We also limited the number of referrals to specialist care in the preceding year to one each day, to avoid duplication arising from counting two Read codes being entered for the same referral.

We identified long term conditions that were recorded in the general practice data between 2001 and 2011 and had not been recorded as resolved. We did not rely on the hospital episode statistics data to assess the long term conditions of individual patients, since in such data, conditions are more likely to be recorded for patients who are admitted frequently. The long term conditions included are the 17 measured as part of the
Quality Outcomes Framework for general practice in England (see table 1).42

\section{Statistical analysis}

We used multivariable generalised linear regression to test the association between the number of admissions for ambulatory care sensitive conditions and the usual provider of care index score, controlling for the covariates. We selected the distribution for our regression model based on an assessment of Pearson residuals. Since over-dispersion was present (and also the variance of the number of admissions for ambulatory care sensitive conditions was greater than the mean), we preferred a negative binomial model (with a log-link) to a Poisson model. ${ }^{43}$ We selected covariates using the Akaike Information Criterion; this supported including all of our chosen covariates. ${ }^{44}$ We expected that two patients in the same practice were likely to have more similar rates of admission than two patients in randomly different practices. Therefore, our model included practice level random effects, but we carried out likelihood ratio tests to determine whether the practice level variation was statistically significant from zero. 4546

We interpreted our model by exponentiating the coefficients to estimate incidence rate ratios for an incremental change in the usual provider of care index score. These incidence rate ratios were then scaled to correspond to an increase in the usual provider of care index score of 0.2around 1 standard deviation-and interpreted as the percentage change in hospital admissions for ambulatory care sensitive conditions. Thus, the incidence rate ratio shows our estimate of the relative change in the number of admissions to result from an increase in the usual provider of care index score of 0.2 , which might occur from any baseline level (for example, from 0.3 to 0.5 or from 0.6 to 0.8 ), and when holding the covariates constant. Since this model assumed a linear relation between the usual provider of care index score and admissions, we also fitted a more flexible model that classified patients as receiving low, medium, and high levels of continuity, rather than treating the usual provider of care index score as a continuous variable. We defined low continuity a priori as a usual provider of care index score between 0 and 0.4 , medium between 0.4 and 0.7 , and high between 0.7 and 1 .

\section{Subgroup analysis}

We conducted subgroup analysis for higher and lower users of general practice care, in each case examining the association between the number of admissions for ambulatory care sensitive conditions and the usual provider of care index score. This was based on dividing the study population into five subgroups of approximately the same size, based on the number of contacts with a general practitioner that each patient experienced over two years. Within each of these subgroups, we estimated the association between the usual provider of care index score and the number of admissions for ambulatory care sensitive conditions using equivalent models to those we have described. In a similar manner, we examined whether the relationship between continuity of care and admissions differed by age group. 


\section{Sensitivity analysis}

Several metrics have been used to measure continuity of care at a patient level. ${ }^{47}$ To ensure our findings were not sensitive to our choice of metric, we additionally performed analysis using the Bice-Boxerman continuity of care index. ${ }^{48}$ Compared with the usual provider of care index, which focuses on the concentration of contacts with a particular general practitioner, the Bice-Boxerman continuity of care index takes into account the dispersion and distribution of contacts a patient has over different general practitioners. ${ }^{49} \mathrm{We}$ re-ran the model predicting admissions to hospital for ambulatory care sensitive conditions including the Bice-Boxerman continuity of care index as an exposure instead of the usual provider of care index. Since the Bice-Boxerman continuity of care index score requires a minimum of three contacts, our analysis was restricted to that subgroup of patients. The Bice-Boxerman continuity of care index score, as with the usual provider of care index score, is bounded between 0 and 1. We also calculated a Pearson correlation coefficient for the two indices, for those patients with three or more contacts.

\section{Patient involvement}

Patients were not involved in setting the research question or the outcome measures, nor were they involved in developing plans for design or implementation of the study. We received valuable feedback on the interpretation and presentation of our results from a patient perspective as part of the review process of The BMJ. As our data are effectively anonymised, we cannot disseminate the results of the research to participants directly, although the Clinical Practice Research Datalink keeps a bibliography of studies published using their data. We plan to disseminate the results to relevant patient communities through alternative mediums, such as blogs and press releases, and by working with national organisations.

\section{Results}

Overall, 263829 patients from 200 general practices met our inclusion and exclusion criteria, of whom $230472 \mathrm{had}$ at least two contacts with general practitioners between April 2011 and March 2013 (again, from 200 practices). On average over the two years, each of these patients experienced 11.40 (SD 9.40) contacts with a general practitioner (table 1). The mean usual provider of care index score was 0.61 (SD 0.23); figure 1 shows the distribution.

The low continuity of care group comprised 52550 patients $(22.8 \%)$, the medium group 96902 patients (42.1\%), and the high group 81020 patients (35.2\%). These groups were similar in terms of their age, sex, and socioeconomic deprivation score (table 1). However, patients in the low continuity group had more contacts with general practitioners on average than the other groups (13.11 per person, compared with 11.34 per person in the medium group and 10.37 in the high group).

Continuity of care as measured by the usual provider of care index tended to be lower in larger practices. In practices with between one and three fulltime equivalent general practitioners, $49.7 \%$ of patients $(n=11883$ ) experienced high continuity of care according to this metric, compared with $30.7 \%$ of patients $(n=34023)$ in practices with seven or more fulltime equivalent

\begin{tabular}{|c|c|c|c|c|}
\hline \multirow[b]{2}{*}{ Variables } & \multicolumn{3}{|l|}{ Continuity of care* } & \multirow{2}{*}{$\begin{array}{l}\text { All patients } \\
(\mathrm{n}=230472)\end{array}$} \\
\hline & Low $(n=52550)$ & Medium ( $\mathrm{n}=96902)$ & High $(n=81020)$ & \\
\hline Women (\% of patients) & 55.77 & 53.95 & 51.88 & 53.63 \\
\hline Mean (SD) age (years) & $71.15(5.88)$ & $71.38(5.87)$ & $71.66(5.87)$ & $71.43(5.88)$ \\
\hline \multicolumn{5}{|l|}{ Socioeconomic deprivation fiftht: } \\
\hline First (least deprived) & $15479(29.46)$ & $26274(27.11)$ & $18906(23.33)$ & $60659(26.32)$ \\
\hline Second & $12360(23.52)$ & $24796(25.59)$ & $21359(26.36)$ & $58515(25.39)$ \\
\hline Third & $9903(18.84)$ & $19603(20.23)$ & $17958(22.16)$ & $47464(20.59)$ \\
\hline Fourth & $8253(15.71)$ & $15733(16.24)$ & $14403(17.78)$ & $38389(16.66)$ \\
\hline Fifth (most deprived) & $6555(12.47)$ & $10496(10.83)$ & $8394(10.36)$ & $25445(11.04)$ \\
\hline Mean (SD) No of long term conditions per patient $¥$ & $1.16(1.18)$ & $1.09(1.14)$ & $1.06(1.14)$ & $1.10(1.14)$ \\
\hline \multicolumn{5}{|l|}{ No of long term conditions per patient:: } \\
\hline 0 & $18345(34.91)$ & $35895(37.04)$ & $30103(37.16)$ & $84343(36.60)$ \\
\hline 1 & 17439 (33.19) & 31915 (32.94) & $27729(34.22)$ & $77083(33.45)$ \\
\hline$\geq 2$ & $16766(31.90)$ & $29092(30.02)$ & $23188(28.62)$ & $69046(29.96)$ \\
\hline Mean (SD) No of contacts with general practitioner over two years per patient & $13.11(10.06)$ & $11.34(9.39)$ & $10.37(8.80)$ & $11.40(9.40)$ \\
\hline Mean (SD) No of referrals to specialist care in 2010/11 per patient & $0.5(0.94)$ & $0.46(0.90)$ & $0.41(0.83)$ & $0.45(0.89)$ \\
\hline Mean (SD) No of admissions for ambulatory care sensitive conditions per patient & $0.18(1.09)$ & $0.15(0.95)$ & $0.14(0.97)$ & $0.16(1.01)$ \\
\hline \multicolumn{5}{|l|}{ No of admissions for ambulatory care sensitive conditions per patient: } \\
\hline 0 & $46314(88.09)$ & $86798(89.53)$ & $73409(90.57)$ & $206521(89.57)$ \\
\hline 1 & $4559(8.67)$ & $7493(7.73)$ & $5705(7.04)$ & $17757(7.70)$ \\
\hline$\geq 2$ & $1702(3.24)$ & $2654(2.74)$ & $1934(2.39)$ & $6290(2.73)$ \\
\hline Mean (SD) No of patients registered at patient's general practice & $11644.64(4004.05)$ & $10958.22(4092.34)$ & $10475.26(4162.45)$ & $10941.41(4124.30)$ \\
\hline Mean (SD) usual provider of care index score & $0.32(0.06)$ & $0.55(0.08)$ & $0.88(0.11)$ & $0.61(0.23)$ \\
\hline
\end{tabular}




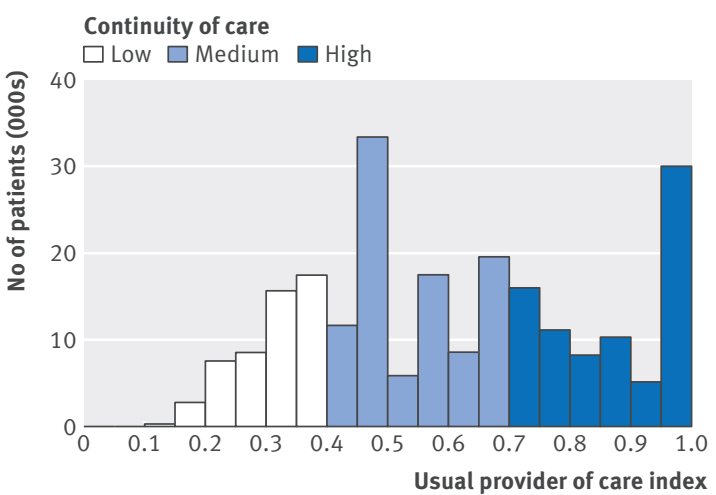

Fig 1 | Distribution of usual provider of care index score across all patients with a minimum of two contacts with general practitioners $(n=230472)$

general practitioners (table 2). As a result, patients with low continuity of care were registered at larger practices than other patients: the average practice list size for patients experiencing low continuity of care was 11644 , compared with 10475 for patients with high continuity of care (table 1).

On average, each patient experienced 0.16 (SD 1.01) admissions for ambulatory care sensitive conditions over the two years, with $89.57 \%$ of patients having no such admission (table 1). After adjusting for the covariates and the usual provider of care index score, results from likelihood ratio tests showed that there remained statistically significant variation between practices in the number of admissions for ambulatory care sensitive conditions. Patients who were older, had more referrals to specialist care, more long term conditions, or more contacts with general practitioners experienced more admissions for ambulatory care sensitive conditions than other patients (table 3). Female sex was associated with having fewer of these admissions, whereas being in the most socioeconomically deprived fifth was associated with having more such admissions.

When adjusting for these covariates, patients with higher continuity of care tended to have fewer admissions for ambulatory care sensitive conditions. Compared with people with low continuity of care, people with medium continuity of care had fewer admissions for ambulatory care sensitive conditions (8.96\%, 95\% confidence interval 5.63\% to $14.22 \%$ ). People with high continuity of care had $12.49 \%$ (9.45\% to $19.29 \%$, table 4) fewer admissions than those with low continuity of care. Overall, across all patients with at least two contacts with a general practitioner, a 0.2 increase in the usual provider of care index score was associated with a reduction in admissions for ambulatory care sensitive conditions of $6.22 \%$ ( $4.87 \%$ to $7.55 \%$, table 5$)$.

\section{Subgroup analysis}

Patients with the highest levels of general practice utilisation tended to have lower continuity of care, while also experiencing more admissions for ambulatory care sensitive conditions. Thus, the heaviest users of general practice had a mean usual provider of care index score of 0.56 (compared with 0.69 for the lowest fifth), and 0.36 admissions on average (compared with 0.04 for the lowest fifth)-see supplementary appendix 2. Table 5 presents the results of the subgroup analysis that examined how the association between the usual provider of care index score and admissions for ambulatory care sensitive conditions varied between fifths defined by general practice utilisation. There was some indication of a U-shaped curve, but the evidence for a link between the usual provider of care index score and admissions was greatest among the most frequent users of general practice. For patients in the two highest fifths of general practice utilisation (12-17 contacts and $\geq 18$ contacts), a 0.2 increase in the usual provider of care index score was associated with a reduction in ambulatory care sensitive admissions of 3.32\% (95\% confidence interval $0.76 \%$ to $5.82 \%)$ and $3.97 \%(1.91 \%$ to $6.00 \%)$, respectively $(\mathrm{P}<0.001)$ (table 5). The associations were less statistically significant at lower levels of general practice utilisation. Table 5 also presents the results of the subgroup analysis for age group: although an inverse relation was observed between the usual provider of care index score and admissions for each age group, the strength of the relationship was larger among older patients.

\section{Sensitivity analysis}

We calculated the Bice-Boxerman continuity of care index score for the 214777 patients who had at least three contacts with general practice in the study period. Among those patients, the Bice-Boxerman continuity of

\begin{tabular}{|c|c|c|c|c|}
\hline Variables & \multicolumn{3}{|c|}{ Practice size (No of FTE* GPs) } & All practices \\
\hline No of practices & 67 & 58 & 40 & 200 \\
\hline \multicolumn{5}{|l|}{ Usual provider of care index for patientst: } \\
\hline \multicolumn{5}{|l|}{ Mean No (\%) of patients by continuity of care: } \\
\hline Low & 30027 (27.08) & $12525(20.26)$ & $2785(11.65)$ & 52550 \\
\hline Medium & $46822(42.23)$ & $26569(42.98)$ & $9247(38.67)$ & 96902 \\
\hline High & $34023(30.69)$ & $22721(36.76)$ & $11883(49.69)$ & 81020 \\
\hline
\end{tabular}




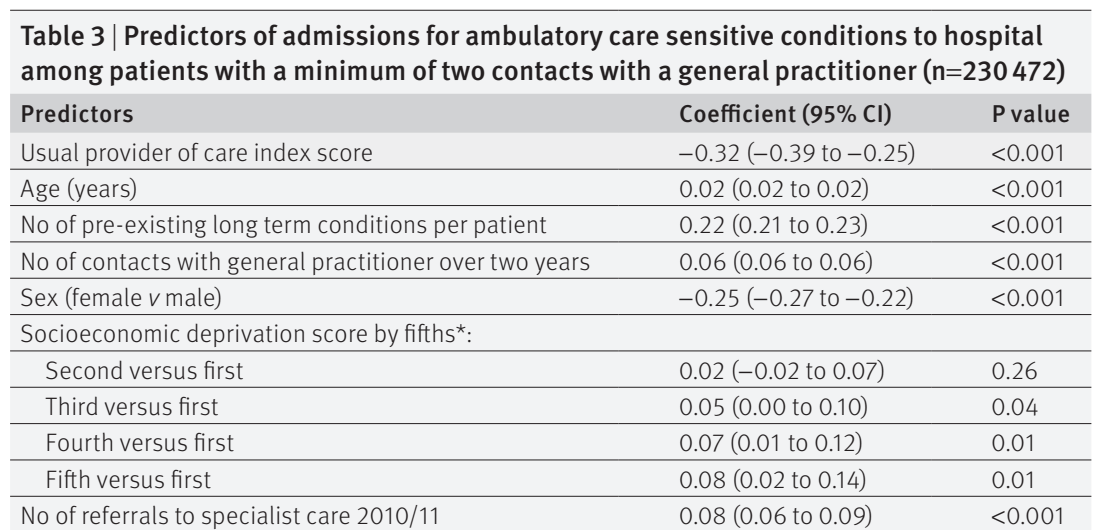

*From first fifth (least deprived) to fifth fifth (most deprived).

In this model, the usual provider of care index score was entered as a continuous variable, with the association with admissions for ambulatory care sensitive conditions estimated using the negative binomial distribution with a log-link. Results can be interpreted by constructing incidence rate ratios for an incremental change in a variable. As the variables have a log-link, those incidence rate ratios can be constructed for a unit change in a covariate by taking the exponential of the coefficients.

Table 4 | Relation between admissions for ambulatory care sensitive conditions and continuity of care ( $n=230472)$

\begin{tabular}{lll} 
& $\begin{array}{l}\text { Relative } \% \text { change in admissions } \\
\text { for ambulatory care sensitive } \\
\text { conditions }(95 \% \mathrm{Cl})\end{array}$ & P value \\
Medium (versus low) & $-8.96(-5.63$ to -14.22$)$ & $<0.001$ \\
\hline High (versus low) & $-12.49(-9.45$ to -19.29$)$ & $<0.001$ \\
\hline High (versus medium) & $-3.87(-0.430$ to -7.19$)$ & 0.03 \\
\hline
\end{tabular}

In this model, the usual provider of care index score was entered as an ordered, categorical variable (with low, medium, and high values), with admissions for ambulatory care sensitive conditions estimated using the negative binomial distribution with a log-link. The model adjusted to control for age, number of pre-existing long term conditions, number of contacts with a general practitioner, sex, socioeconomic deprivation, and number of referrals to specialist care in preceding year.

care index score was strongly correlated with the usual provider of care index score, showing a Pearson correlation coefficient of 0.96 . When we repeated our modelling using the Bice-Boxerman continuity of care index, a 0.2 increase in that score was associated with 3.58\% fewer admissions for ambulatory care sensitive conditions (95\% confidence interval $2.40 \%$ to $4.76 \%, \mathrm{P}<0.001$ ).

\section{Discussion}

Preventing hospital admissions is a priority in many countries, and in England unplanned admissions for ambulatory care sensitive conditions (those considered to be manageable in primary care) accounted for $£ 1.42$ bn of spending in 2009/10 (£170 590 for each general practice). ${ }^{34}$ Since strategies are needed to manage demand for secondary care, we examined the association between continuity of care and hospital admissions among older patients (aged 62-82 years). Continuity of care varied considerably across general practices in England, with $48.6 \%$ of patients in smaller practices and $30.7 \%$ of patients in larger practices falling into our defined high continuity group.

Patients who saw the same general practitioner a greater proportion of the time experienced fewer admissions to hospital for ambulatory care sensitive conditions than other patients. Indeed, compared with patients with low continuity of care, patients with medium continuity of care experienced $8.96 \%$ fewer of these admissions, and those with high continuity of care experienced $12.49 \%$ fewer. There was greater evidence for an association between continuity of care and admissions among those with the highest levels of contacts with general practitioners. As well as showing this clearer link between continuity and reductions, the heaviest users had lower continuity of care (mean usual provider of care index $0.56 v 0.61)$ and more admissions for ambulatory care sensitive conditions ( 0.36 per person on average $v 0.16$ ).

Our findings suggest there is opportunity to improve care and reduce hospital admissions among the highest users of healthcare, but there are various explanations of our findings that must first be carefully considered. One possibility is that when patients receive more continuous care, their doctors might be better able to understand their health needs within time constrained appointments and thus provide care that is more appropriate to the needs of the patient. Continuity of care

\begin{tabular}{|c|c|c|}
\hline Group or subgroup of analysis & $\begin{array}{l}\text { Relative \% change in admissions for } \\
\text { ambulatory care sensitive conditions } \\
(95 \% \text { CI) for a } 0.2 \text { increase in usual } \\
\text { provider of care index score }\end{array}$ & Pvalue \\
\hline All patients $(\mathrm{n}=230472)$ & $--6.22(-7.55$ to -4.87$)$ & $<0.001$ \\
\hline \multicolumn{3}{|l|}{ Fifths of contact (No of contacts): } \\
\hline First $(2-4)(n=49161)$ & $-3.48(-7.65$ to 0.87$)$ & 0.12 \\
\hline Second $(5-7)(n=47208)$ & $-3.89(-7.49$ to -0.14$)$ & 0.04 \\
\hline Third $(8-11)(n=48617)$ & $0.34(-2.59$ to 3.36$)$ & 0.82 \\
\hline Fourth $(12-17)(n=43246)$ & $-3.32(-5.82$ to -0.76$)$ & 0.01 \\
\hline Fifth $(\geq 18)(n=42240)$ & $-3.97(-6.00$ to -1.91$)$ & $<0.001$ \\
\hline \multicolumn{3}{|c|}{ Age group (years) of patients in 2012 with at least two contacts with general practice: } \\
\hline $62-68(n=101761)$ & $-3.40(-5.57$ to -1.19$)$ & 0.003 \\
\hline $69-75(n=74161)$ & $-7.92(-10.22$ to -5.56$)$ & $<0.001$ \\
\hline $76-82(n=54550)$ & $-8.37(-10.7$ to -5.99$)$ & $<0.001$ \\
\hline \multicolumn{3}{|c|}{$\begin{array}{l}\text { In these models, the usual provider of care index score was entered as a continuous variable, with admissions for ambulatory care sensitive conditions } \\
\text { using the negative binomial distribution with a log-link. Results were interpreted by constructing incidence rate ratios for an incremental change in usual } \\
\text { provider of care index score. As the variables have a log-link, incidence rate ratios for a unit change in a covariate were constructed by taking the } \\
\text { exponential of the coefficients. The results are interpreted for a } 0.2 \text { change of the usual provider of care index score. Each model is adjusted to control for } \\
\text { age, number of pre-existing long term conditions, number of contacts with a general practice, sex, socioeconomic deprivation, and number of referrals } \\
\text { to specialist care in preceding year. }\end{array}$} \\
\hline
\end{tabular}


might also promote a more effective and trusting relationship between patients and doctors, leading to a better understanding of health problems and greater adherence to the agreed course of treatment. One would expect these benefits to be felt most keenly in those patients who need to visit the general practitioner frequently. However, it is important to note that identifying a causal mechanism is beyond the scope of this study.

To our knowledge no previous study has examined the association between longitudinal continuity of care in primary care (measured by usual provider of care index) and admissions for ambulatory care sensitive conditions. However, a study of older patients in primary care in one Canadian province found that those with a medium degree of longitudinal continuity of care, measured through the usual provider of care index, had $27 \%$ (95\% confidence interval $25 \%$ to $29 \%$ ) more visits to an emergency department than those with high continuity of care. ${ }^{26}$ And although it relates to a different process, the GP patient survey in England asks patients whether they were able to see the general practitioner of their choice, and practices scoring more highly on this metric tend to have lower rates of hospital admissions. ${ }^{24}$ A body of literature has looked at the effect of continuity in secondary care..$^{50} 51$

\section{Strengths and limitations of this study}

We were able to study linked records for 230472 patients, resulting in small confidence intervals around our estimated values. Patients registered with practices participating in the Clinical Practice Research Datalink are broadly representative of those in England, but we restricted our analysis to practices submitting up-to-standard data. ${ }^{40}$ We examined the period between 2011 and 2013; however, we do not believe that the associations found are likely to have changed with time. We cannot directly assess the representativeness of the 200 practices in our study sample, although we note that $26.3 \%$ of our study sample lived in the $20 \%$ least socioeconomically deprived areas, compared with $11.0 \%$ in the $20 \%$ most deprived areas. This may be partly because our study was restricted to those aged between 62 and 82 years. We focused on older patients because they tend to experience more admissions for ambulatory care sensitive conditions than other age groups, ${ }^{34}$ but older people may be less likely to live in deprived areas. ${ }^{52}$ In addition, we limited our analysis to those patients with a minimum of two contacts with general practitioners, to ensure our metric was sensitive. Although we cannot establish the association between the usual provider of care index score and admissions for people younger than 62 or older than 82 , we note that our subgroup analysis showed a strong age gradient within our sample. More studies are needed, but it is possible that continuity of care is more directly related to admissions among those with a higher burden of chronic disease, which includes older people as well as those living in more deprived areas.

We chose the usual provider of care index since it is more easily interpreted than some of the other metrics of care continuity. ${ }^{4749}$ However, we performed sensitivity analysis using the Bice-Boxerman continuity of care index, which compared with the usual provider of care index is more sensitive to the distribution of contacts across multiple doctors (in addition to the proportion of contacts with most regular doctors). ${ }^{48}$ The two indices were highly correlated. Although the level of association differed, both showed an association with admissions for ambulatory care sensitive conditions. Furthermore, the association remained when we modelled the usual provider of care index score as a categorical rather than continuous variable. In that analysis, the thresholds we applied to divide patients into high, medium, and low continuity of care groups were necessarily arbitrary, and there were also some limitations to the way in which we calculated the usual provider of care index score. By focusing on general practitioners, we did not assess the importance of the continuity of care provided by practice nurses and healthcare assistants. Also, some caution is needed in relation to larger practices, since it is possible that in those practices patients are deliberately managed by several general practitioners working in a small team (for example, to provide cover when doctors work part time). Ideally, we would have adapted the usual provider of care index to allow for these "microteams," but the requisite information was not available.

We aimed to assess longitudinal continuity of care, and we could not assess the quality of the interactions from the patient perspective or whether patients saw their preferred doctor. It is possible that measures of interpersonal, management, and informational continuity would also show an association with utilisation of secondary care, and that patients' definitions of continuity of care might vary from that presented here. ${ }^{13} \mathrm{By}$ analysing admissions for ambulatory care sensitive conditions, rather than all unplanned admissions, we aimed to focus on those admissions most likely to be preventable by good quality primary care. However, administrative data provide limited information to determine whether individual admissions are avoidable or desirable. For example, heart failure is often classified as an ambulatory care sensitive condition, but even when managed according to evidence informed guidelines, it will gradually deteriorate to the point at which hospital admission may be required. However, if only a subset of the admissions for ambulatory care sensitive conditions were avoidable, then this would bias the magnitude of our results towards showing a smaller association. We did not examine the association between continuity of care and other forms of secondary care, community healthcare, or social care, and indeed there is some evidence that increased continuity of care may lead to later referral for cancer care. ${ }^{31}$

This was a cross sectional, observational study. Although such studies can test for associations between characteristics, a common concern is that any association may be attributable to differences in unobserved confounders. Our analysis cannot tease out whether continuity is a component of high quality care that has a consequential effect on hospital admissions, or whether increased continuity of care and reduced hospital admissions are both outcomes of high quality care. Furthermore, a cross sectional study cannot examine the 
mechanism or direction of action-for example, general practitioners may alter their management of a patient immediately before or after an admission to hospital, potentially leading to improved continuity of care after hospital discharge. Moreover, although we examined patient subgroups defined by general practice utilisation, continuity of care might be particularly important for groups that we could not isolate from the data, such as those unable to adopt self care strategies..$^{53}$ Continuity of care might also be more important at some times than others-for example, according to the onset of acute health problems or after discharge from hospital. Finally, the importance of continuity of care might vary according to local context and the broader approach to managing patients within a general practice, including the role of practice nurses and specialist general practitioners, how information is shared within the practice, and working in a micro-team.

\section{Conclusions and implications}

This study motivates a renewed focus on promoting continuity of care, and it suggests that continuity is an important consideration when designing approaches to reduce hospital admissions. Over the past decade, initiatives to prevent hospital admissions have tended to prioritise speed and equity of access to primary care over continuity of care. ${ }^{54}$ In England, one of the few recent national initiatives to improve the continuity of care in general practice was the mandatory introduction of named accountable general practitioners, ${ }^{55}$ but this had no discernible impact on longitudinal continuity of care, at least for patients aged 75 over the first nine months. ${ }^{56}$ Therefore, approaches are needed to improve continuity of care in general practice.

Several approaches have been suggested, including increasing patient awareness of the importance of continuity, changing receptionists' behaviour or practice booking systems to promote continuity, and organising large practices into small teams, each of which care for a subset of the patients registered at the practice, meaning that patients receive continuous care at team level rather than individual level. These can be explored further. ${ }^{9}$ Of course it cannot be assumed on the basis of this study that interventions that successfully improve the continuity of care will necessarily lead to reductions in hospital admissions for ambulatory care sensitive conditions, and interventions will require careful evaluation. A complementary approach would be to make data on continuity of care available to general practice teams for quality improvement initiatives, as recommended by the Royal College of General Practitioners. ${ }^{57}$ In this study we measured continuity of care using data that are already available to general practice teams from the electronic medical record, although in some cases the analytical capability to analyse these data may be lacking. ${ }^{58}$ Despite the limitations of these data, we have demonstrated a link with hospital admission rates. We have also shown that interventions could be targeted at easily identifiable groups of patients. This suggests that the usual provider of care index could inform strategies for quality improvement, although it may need to be adapted for local context, and its responsiveness to service change would need to be tested.

Continuity of care aligns closely with the reasons many people chose to work in general practice, ${ }^{15}$ is important to patients, ${ }^{29}$ correlates with outcomes, ${ }^{1216242659}$ and shows variability between general practices. As we show, continuity of care is also associated with avoidable hospital admissions. Thus initiatives that improve the continuity of care have the potential to improve the quality of healthcare while reducing cost, both fundamental aims of healthcare systems.

We thank Therese Lloyd for helping to prepare the dataset for analysis, sharing code, and methodological advice; Will Warburton and Martin Roland for their helpful and insightful comments on an earlier version of this manuscript; and the four peer reviewers for feedback, which helped us to improve the manuscript. This study is based in part on data from the Clinical Practice Research Datalink obtained under license from the UK Medicines and Healthcare products Regulatory Agency. However, the interpretation and conclusions contained in this study are those of the authors alone.

Contributors: IB, AS, and SD had the idea for the study and submitted a major amendment to a previous Clinical Practice Research Datalink application to facilitate the research. IB, AS, and SD came up with the statistical analysis plan. IB and SD carried out the analysis. IB, SD, and AS drafted and finalised the paper. IB is the guarantor.

Funding: None received.

Competing interests: All authors have completed the ICMJE uniform disclosure form at www.icmje.org/coi_disclosure.pdf and declare: all authors had financial support from The Health Foundation for the submitted work; no financial relationships with any organisations that might have an interest in the submitted work in the previous three years; no other relationships or activities that could appear to have influenced the submitted work.

Ethical approval: This study was approved by the Clinical Practice Research Datalink independent scientific advisory committee. This was secondary analysis of data submitted to the Clinical Practice Research Datalink, no patient consent forms were required to access this dataset.

Data sharing: The data controller of the data analysed is the Clinical Practice Research Datalink. Patient level data are available subject to their information governance requirements.

Transparency: The lead author (IB) affirms that the manuscript is an honest, accurate, and transparent account of the study being reported; that no important aspects of the study have been omitted; and that any discrepancies from the study as planned (and, if relevant, registered) have been explained.

This is an Open Access article distributed in accordance with the Creative Commons Attribution Non Commercial (CC BY-NC 3.0) license, which permits others to distribute, remix, adapt, build upon this work non-commercially, and license their derivative works on different terms, provided the original work is properly cited and the use is non-commercial. See: http://creativecommons.org/licenses/ by-nc/3.0/.

1 Huntley A, Lasserson D, Wye L, et al. Which features of primary care affect unscheduled secondary care use? A systematic review. BMJ Open 2014:4:e004746. doi:10.1136/bmjopen-2013-004746.

2 Roland M, Abel G. Reducing emergency admissions: are we on the right track?BMJ 2012;345:e6017. doi:10.1136/bmj.e6017.

NHS England. Five Year Forward View. www.england.nhs.uk/ wp-content/uploads/2014/10/5yfv-web.pdf.

4 Whittington JW, Nolan K, Lewis N, Torres T. Pursuing the Triple Aim: The First 7 Years. Milbank Q 2015;93:263-300. doi:10.1111/1468-0009.12122.

5 Cowling TE, Cecil EV, Soljak MA, et al. Access to primary care and visits to emergency departments in England: a cross-sectional, population-based study. PLoS One 2013;8:6-11. doi:10.1371/journal.pone.0066699.

6 Osborn R, Moulds D, Schneider EC, Doty MM, Squires D, Sarnak DO Primary care physicians in ten countries report challenges caring for patients with complex health needs. Health Aff (Millwood) 2015;34:2104-12. doi:10.1377/hlthaff.2015.1018.

Edes T, Kinosian B, Vuckovic NH, Nichols LO, Becker MM, Hossain M. Better access, quality, and cost for clinically complex veterans with home-based primary care. J Am Geriatr Soc 2014;62:1954-61. http://onlinelibrary.wiley. com/doi/10.1111/jgs.13030/full. doi:10.1111/.jgs.13030. 
8 Henderson C, Knapp M, Fernández J-L, et al. Whole System Demonstrator evaluation team. Cost effectiveness of telehealth for patients with long term conditions (Whole Systems Demonstrator telehealth questionnaire study): nested economic evaluation in a pragmatic, cluster randomised controlled trial. BMJ 2013;346:f1035. doi:10.1136/bmj.f1035.

9 Roland M. Continuity of care: betrayed values or misplaced nostalgia. Int J Integr Care 2012;12:e200. doi:10.5334/ijic.1056.

10 Katz DA, McCoy KD, Vaughan-Sarrazin MS. Does Greater Continuity of Veterans Administration Primary Care Reduce Emergency Department Visits and Hospitalization in Older Veterans?) Am Geriatr Soc 2015;63:2510-8. doi:10.1111/jgs.13841.

11 Institute of Medicine. Crossing the quality chasm: a new health system for the 21st century. 2001. www.nap.edu/read/10027/chapter/1\#il.

12 Saultz JW, Albedaiwi W. Interpersonal continuity of care and patient satisfaction: a critical review. Ann Fam Med 2004;2:445-51. doi:10.1370/afm.91.

13 Adler R, Vasiliadis A, Bickell N. The relationship between continuity and patient satisfaction: a systematic review. Fam Pract 2010;27:171 8. doi:10.1093/fampra/cmp099.

14 Chen H-M, Tu Y-H, Chen C-M. Effect of continuity of care on quality of life in older adults with chronic diseases: a meta-analysis. Clin Nurs Res 2016; Jan 20. doi:10.1177/1054773815625467.

15 Kassler WJ, Wartman SA, Silliman RA. Why medical students choose primary care careers. Acad Med 1991;66:41-3. www.scopus.com/ inward/record.url?eid=2-s2.0-0026065158\&partnerlD=40\&md5=40 994f8badc82b1726b1541d4657911e. doi:10.1097/00001888-199101000-00012.

16 National Survey of GPs. The Future of General Practice 2015. BMA, 2015. http://bma.org.uk/working-for-change/negotiating-for-the-profession/ bma-general-practitioners-committee/surveys/future-of-general-practice.

17 Ipsos MORI. GP Patient Survey - National summary report: January 2015 publication. 2015. https://gp-patient.co.uk/surveys-and-reports

18 Baird B, Charles A, Honeyman M, et al. Understanding pressures in general practice. King's Fund, 2016.

19 Ladapo J, Chokshi D. Continuity Of Care For Chronic Conditions: Threats, Opportunities, And Policy. Health Affairs Blog, 2014.

20 Marshall M, Gray DP. General practice is making a leap in the dark BMJ 2016;355:i5698. doi:10.1136/bmj.i5698.

21 Campbell SM, Hann M, Hacker J, et al. Identifying predictors of high quality care in English general practice: observational study. BMJ 2001:323:784-7. doi:10.1136/bmj.323.7316.784.

22 Campbell JL, Ramsay J, Green J. Practice size: impact on consultation length, workload, and patient assessment of care. Br J Gen Pract 2001;51:644-50.

23 Baker R, Streatfield J. What type of general practice do patients prefer? Exploration of practice characteristics influencing patient satisfaction. BrJ Gen Pract 1995;45:654-9.

24 Bankart MJG, Baker R, Rashid A, et al. Characteristics of general practices associated with emergency admission rates to hospital: a cross-sectional study. Emerg Med J 2011;28:558-63. doi:10.1136/emj.2010.108548.

25 Clark WAV, Avery KL. The effects of data aggregation in statistical analysis. Geogr Anal 1976;VIII:428-38. http://onlinelibrary.wiley.com/ doi/10.1111/gean.1976.8.issue-4/issuetoc.

26 Ionescu-Ittu R, McCusker J, Ciampi A, et al. Continuity of primary care and emergency department utilization among elderly people. CMAJ 2007;177:1362-8. doi:10.1503/cmaj.061615.

27 Rosenblatt RA, Wright GE, Baldwin L, et al. The effect of the doctor - patient relationship on emergency department use among the elderly. Am J Public Health 2000;90:97-102doi:10.2105/AJPH.90.1.97

28 Gunther S, Taub N, Rogers S, Baker R. What aspects of primary care predict emergency admission rates? A cross sectional study. BMC Health Serv Res 2013;13:11. doi:10.1186/1472-6963-13-11.

29 Saultz JW, Lochner J. Interpersonal continuity of care and care outcomes: a critical review. Ann Fam Med 2005;3:159-66. doi:10.1370/afm.285.

30 Guthrie B, Wyke S. Personal continuity and access in UK general practice: a qualitative study of general practitioners' and patients' perceptions of when and how they matter. BMC Fam Pract 2006;7:11 doi:10.1186/1471-2296-7-11.

31 Ridd MJ, Ferreira DL, Montgomery AA, Salisbury C, Hamilton W. Patient-doctor continuity and diagnosis of cancer: electronic medical records study in general practice. Br J Gen Pract 2015;65:e305-11. doi:10.3399/bigp15X684829.

32 Bardsley M, Blunt I, Davies S, Dixon J. Is secondary preventive care improving? Observational study of 10 -year trends in emergency admissions for conditions amenable to ambulatory care. BMJ Open 2013;3:1-12. doi:10.1136/bmjopen-2012-002007.

33 Billings J, Zeitel L, Lukomnik J, Carey TS, Blank AE, Newman L. DataWatch: Impact of socioeconomic status on hospital use in New York City. Health Aff 1993;12:162-73. doi:10.1377/hlthaff.12.1.162

34 Tian Y, Dixon A, Gao H. Data briefing - Emergency hospital admissions for ambulatory care-sensitive conditions: identifying the potential for reductions. King's Fund, 2012.

35 Cheng S-H, Chen C-C, Hou Y-F. A longitudinal examination of continuity of care and avoidable hospitalization: evidence from a universal coverage health care system. Arch Intern Med 2010:170:1671-7. doi:10.1001/archinternmed.2010.340.
36 Lin W, Huang IC, Wang S-L, Yang MC, Yaung CL. Continuity of diabetes care is associated with avoidable hospitalizations: evidence from Taiwan's National Health Insurance scheme. Int I Qual Health Care 2010;22:3-8. doi:10.1093/intqhc/mzp059.

37 Tsai H-Y, Chou Y-J, Pu C. Continuity of care trajectories and emergency room use among patients with diabetes. Int J Public Health 2015:60:505-13 doi:10.1007/s00038-015-0671-1.

38 Reddy A, Pollack CE, Asch DA, Canamucio A, Werner RM. The Effect of Primary Care Provider Turnover on Patient Experience of Care and Ambulatory Quality of Care. JAMA Intern Med 2015;175:1157-62. doi:10.1001/jamainternmed.2015.1853.

39 Lloyd T, Steventon A. Effect of Named, Accountable GPs on Continuity of Care: Protocol for a Regression Discontinuity Study of a National Policy Change. Int J Integr Care 2016;16:6. doi:10.5334/ijic.2450.

40 Herrett E, Gallagher AM, Bhaskaran K, et al. Data Resource Profile: Clinical Practice Research Datalink (CPRD). Int J Epidemiol 2015:44:827-36. doi:10.1093/ije/dyv098.

41 Office for National Statistics. Atlas of Deprivation 2010. 2010. www. ons.gov.uk/ons/rel/regional-trends/atlas-of-deprivation-england/2010/atlas-of-deprivation-2010.html (accessed 7 Oct 2015).

42 Employers NHS. 2014/15 General Medical Services (GMS) Contract Quality and Outcomes Framework (QOF). Published Online First: 2014. www.nhsemployers.org/your-workforce/primary-care-contacts / general-medical-services/quality-and-outcomes-framework.

43 Hilbe JM. Negative Binomial Regression, 2nd edn. Cambridge University Press, 2011doi:10.1017/CB09780511973420.

44 Akaike H. A new look at the statistical model identification. IEEE Trans Automat Contr 1974;19:716-23. doi:10.1109/TAC.1974.1100705.

45 Stroup WW. Generalized Linear Mixed Models: Modern Concepts, Methods and Applications. CRC Press, 2012

46 Singer JD. Using SAS PROC MIXED to Fit Multilevel Models, Hierarchical Models, and Individual Growth Models. J Educ Behav Stat 1998;23:323-55. doi:10.2307/1165280.

47 Jee SH, Cabana MD. Indices for continuity of care: a systematic review of the literature. Med Care Res Rev 2006;63:158-88. doi:10.1177/1077558705285294.

48 Bice TW, Boxerman SB. A quantitative measure of continuity of care. Med Care 1977:15:347-9. doi:10.1097/00005650-197704000-00010.

49 Salisbury C, Sampson F, Ridd M, Montgomery AA. How should continuity of care in primary health care be assessed?Br J Gen Pract 2009:59:e134-41. doi:10.3399/bigp09X420257.

50 Poteliakhoff JCRLLSE. Continuity of care for older hospital patients. King's fund 2012;1-32. www.kingsfund.org.uk/sites/files/kf/field/ field publication file/continuity-of-care-for-older-hospital-patientsmar-2012.pdf\%5Cnpapers2://publication/uuid/ C7B80A53-BB20-4838-8742-6DD73BAED196

51 Blecker S, Shine D, Park N, et alAssociation of weekend continuity of care with hospital length of stay. Int I Qual Health Care 2014:26:5307doi:10.1093/intqhe/mzu065

52 Charlton J, Rudisill C, Bhattarai N, Gulliford M. Impact of deprivation on occurrence, outcomes and health care costs of people with multiple morbidity. J Health Serv Res Policy 2013;18:215-23. doi:10.1177/1355819613493772

53 Hibbard JH, Mahoney ER, Stock R, Tusler M. Do increases in patient activation result in improved self-management behaviors? Health Serv Res 2007;42:1443-63. doi:10.1111/j.1475-6773.2006.00669.x

54 Royal College of General Practitioners. Continuity of care in general practice. 2016. www.rcgp.org.uk/-/media/Files/Policy/A-Z-policy/2016/ Continuity-of-care-in-modern-day-general-practice1.ashx?la=en

55 Department of Health. Transforming Primary Care. London: Department of Health 2014. www.gov.uk/government/uploads/ system/uploads/attachment_data/file/304139/Transforming_ primary_care.pdf (accessed 10 May 2015).

56 Barker I, Lloyd T, Steventon A. Effect of a national requirement to introduce named accountable general practitioners for patients aged 75 or older in England: regression discontinuity analysis of general practice utilisation and continuity of care. BMJ Open 2016;6:e011422 doi:10.1136/bmjopen-2016-011422

57 Royal College of General Practitioners. RCGP Continuity of Care Toolkit. Royal College of General Practitioners 2012. www.rcgp.org.uk/policy/ rcgp-policy-areas/ /media/Files/Policy/A-Z-policy/Continuity\%20 of\%20Care\%20Toolkit.ashx

58 Bardsley M. Understanding analytical capability in health care. 2016. www.health.org.uk/sites/health/files/

UnderstandingAnalyticalCapabilitylnHealthCare.pdf

59 Freeman GK, Olesen F, Hjortdahl P. Continuity of care: an essential element of modern general practice?Fam Pract 2003;20:623-7. doi:10.1093/fampra/cmg601

Supplementary appendix 1: Ambulatory care sensitive conditions and related ICD-10 codes Supplementary appendix 2: Characteristics of patient subgroups defined by fifths of contact with a general practitioner over two years 